\title{
Acknowledgment of Ad Hoc Reviewers, 2016
}

\section{Principal Ad Hoc Reviewers}

The Editor and Associate Editors wish to acknowledge the following ad hoc reviewers. These individuals reviewed four or more manuscripts for the Journal of Occupational Health Psychology during the period between July 2015 through June 2016 and were designated Principal Ad Hoc Reviewers in recognition of their service.

Jari J. Hakanen

Pascale M. Le Blanc
Joseph Nicholas Luchman

YoungAh Park
Ana Isabel Sanz-Vergel

Antje Schmitt

Dieter Zapf

Ad Hoc Reviewers

Gary A. Adams

Jef Adriaenssens

Jos Akkermans

Muhammad Ali

Joseph Allen

Toni Alterman

Kara A. Arnold

Richard Backs

Peter A. Bamberger

Larissa K. Barber

Julian Barling

Brenda L. Bass

Harry Becher*

Myriam N. Bechtoldt

Misty Bennett

Mindy Bergman

Miguel Bernabe

Claudia Bernhard-Oettel

Jeremy M. Beus

Caroline Biron

Petri Bockerman

Prashant Bordia

Nathan A. Bowling

Lisa Bradley

Jill Bradley-Geist

Thomas W. Britt

Paula A. Brough

Valentina Bruk-Lee

Carrie A. Bulger

Jennifer Bunk

James Burton

Gabriele Buruck

Lori A. Button

Orfeu M. Buxton

Roman Cieslak

Eva Cifre-Gallego

Konstantin P. Cigularov

Sharon Clarke

Jeanette Cleveland

Michel Cossette

Mark Cropley

Dev Dalal

Kevin Daniels

Kelly D. Davis

Timothy DeGroot

Jan de Jonge

Irene de Pater
Daantje Derks

Jan Dettmers

Stefan Diestel

Lee Di Milia

Maureen Dollard

Kathryne E. Dupre

Erin Eatough

Achim Elfering

Kevin Eschleman

Jinyan Fan

Jorg Felfe

Mark T. Fleming

Mark C. Frame

Franziska Franke

Desiree Fullemann

Elisabeth Gilbert*

Stephanie Gilbert

Nicolas Gillet

Gary W. Giumetti

Sharon Glazer

Gunne Grankvist

Maja Graso

Nicole Gravina

Matthew J. Grawitch

Yannick Griep

James Grosch

Jari J. Hakanen

Peter M. Hart

Steve Harvey

Jan A. Hausser

Hilde Hetland

Rebecca Hewett

Jeffrey S. Hickman

Ivona Hideg

Lisle S. Hites

Severin Hornung

Ute Hulsheger

Paul Jansen

Gary Johns

Dana Kabat-Farr

Seth Kaplan

Maria Karanika-Murray

Leila Karimi

Stacey Kessler
Sanna Kinnunen

Ulla Kinnunen

Dejun Tony Kong

Ben Kuo

Pascale M. Le Blanc

Jin Lee

Marcie LePine

Chang-qin Lu

Joseph Nicholas Luchman

Aleksandra Luksyte

Gil Luria

Jeremy Mackey

Jay A. Mancini

Aaron Manier

Janet Mantler

Sophia Marinova

Frances M. McKee-Ryan

Nicholas S. Miceli

Valerie Morganson

Jane Mullen

Katharina Naswall

Alexander Newman

Karina Nielsen

Cornelia Niessen

Heather Odle-Dusseau

Michael P. O'Driscoll

Debra Gilin Oore

Alayne Ormerod

Katie M. Page

YoungAh Park

Kelsey Parker

Samantha C. Paustian-

Underdahl

Maria Peeters

Yisheng Peng

Sara Jansen Perry

Paraskevas Petrou

Anja Philipp

Sabine Raeder

Jennifer Ragsdale

Jana Raver

Dorota Reis

Simon Restubog

Jessica Beth Rodell
Lara Christina Roll

Cort W. Rudolph

Ann Marie Ryan

Erika L. Sabbath

Kenji Sakurai

Ana Isabel Sanz-Vergel

John M. Schaubroeck

Klaus-Helmut Schmidt

Antje Schmitt

Winny Shen

Nancy Sin*

Janne Skakon

Michae T. Sliter

Brandon Smit

Simon Smith

Yifan Song*

Florencia M. Sortheix

Karoline Strauss

Naomi Swanson

Sylvi Thun

Maria Tims

Emile Tompa

Louise Tourigny

Thomas M. Tripp

Donald M. Truxillo

Tim Vahle-Hinz

Bob Vandenberg

Marc van Veldhoven

Sabrina DeeAnn Volpone

David Wagner

Benjamin Walsh

Hai-jiang Wang

Julie Holliday Wayne

Jennica Webster

Jennifer H. K. Wong

Stephen Wood

Thomas J. Zagenczyk

Dieter Zapf

Christopher Zatzick

Yujie Zhan

Xinyuan Zhao

Le Zhou

Zhiqing E. Zhou

Fred R. H. Zijlstra

Ina Zwingmann

* Denotes a co-reviewer supervised by a primary reviewer. 